bodies, the meetings of which its members attend. Members of the University of London have also paid visits in connexion with the development of the scheme of 'special relationships'. This frequent intercourse with the home universities has proved invaluable in assisting the progress of the colleges, smoothing out difficulties, promoting co-operation and removing the sense of isolation. A further important service has been rendered by the InterUniversity Council by its appointment of a library advisor, whose office in London has already become an important centre of information and advice. In the preparation of book-lists, the supply of information on micro-films and other technical matters, procuring sets of periodicals, advice on buildings and furniture, on binderies and library organisation, much help is asked for and given.

One of the most difficult and pressing problems experienced by all the new colleges is naturally that of staffing. The ultimate success of these new institutions will depend predominantly on the quality of the staff appointed, particularly in the early stages of their existence. The Inter-University Council, with the active co-operation of the home universities, has expended much effort in meeting the demand. A large measure of success has attended this work, though there are many important posts still unfilled. It is good to know that the policy of the Council is not to fill a post at all rather than to appoint an individual not up to the necessary university standard in teaching and research ability. It had been hoped to make a considerable number of appointments by secondment for limited periods up to three years of members of home universities. A few such have been made, but there is room for many more. Such appointments, it may be remarked, should benefit in the long run the seconding as much as the receiving universities.

A welcome start has been made in associating research institutes with the nascent universities in accordance with the policy of the Colonial Research Committee. Institutes of Economic and Social Research have been set up at the University College of the West Indieg, at Mbadan and at Makerere College. Departments of extra-mural studies have also been established in the West Indies, the Gold Coast and Nigeria. These should have a great influence in spreading enlightenment throughout all social classes in the territories and do much to hasten their general progress.

All these developments are, of course, costing much money. The distribution by way of block grants by the Colonial University Grants Advisory Committee of $£ 6$ millions provided by the Colonial Development and Welfare Fund, the generous grants made both for current and capital expenditure by the local governments conoerned, and the further direct financial support, often on a large scale, by the Colonial peoples themselves, the establishment of scholarships by local authorities and private associations, and numerous benefactions both in money and kind, all would appear to ensure a satisfactory financial future.

There can be no doubt that, though there must be difficulties and teething-troubles ahead, yet in the words of the report, "something has come alive in the Colonies in these past two years on which the hopes of many people rest". The Inter-University Council may be well satisfied by the part it has played in this highly significant development.

$$
\text { B. Modat Jones }
$$

\section{EDINBURGH CONFERENCE ON ELEMENTARY PARTICLES}

THE Department of Natural Philosophy of the University of Edinburgh arranged a conference on elementary particles, to take advantage of the presence in Scotland of Prof. Niels Bohr, who has given this year's Gifford Lectures. The conference took place during November 14-16, with a full programme of lectures both experimental and theoretical. In this brief report of the proceedings it will not be possible to do justice to all of the speakers taking part.

In the experimental section of the programme, Prof. N. Feather, of Edinburgh, described various attempts to detect the presence either of negatively charged protons or of neutral particles (dineutrons) of double the mass of a neutron. It was considered that if such particles exist at all in Nature, then one might expect to find them among the radiations emitted in fission or by short-lived fission products. A typical experiment was one carried out by Fenning at Harwell, exposing bismuth to an intense neutron flux in a pile. In this case neutron and dineutron capture will produce beta- and alpha-activity respectively, and the conditions are favourable for detecting the alpha-activity even in very small intensity. The results of this and other experiments have been entirely negative. Dr. B. Zajac and Dr. M. A. S. Ross described experimentel work which is in progress at Edinburgh in the field of cosmic rays, using the photographic plate technique. They have developed an accurate and rapid method of calibra. tion to determine the relation between grain density and specific ionization in a particular plate; they use for this purpose the tracks of electrons which come to rest in the emulsion.

Five other experimental talks were given dealing with cosmic rays: by Dr. J. G. Wilson of Manchester, Dr. B. Pontecorvo of Harwell, Dr. D. H. Perkins of Imperial College, London, Prof. LePrince Ringuet of Paris and Prof. C. F. Powell of Bristol. Dr. Perkins has studied the heavier fragments which are emitted in stars in photographic plates. The statistics are not yet good enough for trustworthy conclusions to be drawn, but it appears that a surprisingly large proportion of large fragments are emitted with relatively high velocities, the average velocity increasing with the charge of the fragment. It is difficult to understand how fragments come to be emitted with such high kinetic energies, and yet with internal excitation energies which are not great enough to cause complete disruption. Dr. Perkins suggests that the explanation may be found in the very high angular momenta which may be given to a heavy nucleus by the impact of a relativistic nucleon striking to one side of the centre of gravity. If the residual nucleus is left with a moderately large ex. citation energy, and with 20 or 30 units of angular momentum, then the emission of fast heavy frag ments may be favoured as the most effective means of disposing of the angular momentum.

Prof. LePrince Ringuet exhibited some slides showing giant stars, in which twenty or thirty lightly ionizing tracks are seen emerging in a bundle from one side of a nuclear disintegration. Such events are prima facie evidence of the multiple production of mesons in a single act. However, after some dis. cussion, it was agreed that the evidence is not conclusive, and an interpretation in terms of plural 
(successive) production of mesons in many individual nucleon-nucleon encounters inside the nucleus is still possible. In this connexion, Prof. L. Jánossy of Dublin presented an analysis of some experimental data obtained at Bristol, consisting of some two hundred pictures of stars with more than two lightly ionizing tracks. He showed that the experimental statistics of such tracks, including three cases of stars with more than sixteen light tracks, can be well accounted for by a hypothesis of purely plural production, using a phenomenological theory with a reasonable assumed cross-section for the production of a single meson in a single nucleon-nucleon collision. It appears that the issue of plural versus multiple production will only be decisively settled by experiments involving the production of mesons in hydrogen. In conclusion, Prof. LePrince Ringuet described an experiment to measure the masses of fast penetrating particles with a mass-spectrometer consisting of a cloud-chamber in a magnetic field combined with a suitable arrangement of absorbing plates and counters, similar to the apparatus of Alichanov, Alichanian and Weissenberg. He found no evidence for masses different from those of the well-known types of particle. He observed that protons which suffer nuclear collisions in the absorber might easily be interpreted as 'varytrons'.

Prof. C. F. Powell presented a more extensive analysis of the masses of fast particles, carried out at Bristol and based on a large number of exposures of minimum-ionization-sensitive plates at various altitudes. Two methods of mass-determination have been used, giving results which are generally in agreement. First, the small-angle scattering has been accurately measured for all tracks having a length of more than $3 \mathrm{~mm}$. in the emulsion; combined with specific ionization measurements, this determines the masses of particlos at non-relativistic velocities, and sets an upper limit for the masses at relativistic velocities. Secondly, Franzinetti has made observations at the Jungfraujoch with two emulsions facing each other and separated by an air-gap, the whole assembly being maintained in a magnetic field of 30,000 gauss; the deflexion of a particle in this field as it crosses the air-gap, together with its range in the emulsion, yields a measure of its mass. The results of these experiments may be summarized as follows. (i) Of all fast tracks, 98 per cent are identified as having masses corresponding to known particles. The remaining 2 per cent, which might be interpreted as $\tau$-mesons, should probably be attributed to the effects of large-angle scattering or other instrumental errors. No new examples have been found of the peculiar types of disintegration which gave rise to the original postulation of the $\tau$-meson. (ii) Let a 'shower particle' be defined as a particle coming out of a star and giving a track with less than one and a half times minimum ionization. Then at least 80 per cent of shower particles are $\pi$-mesons. Also, a considerable proportion of the fast ionizing particles seen entering and presumably causing stars are $\pi$-mesons. (iii) Of the fast tracks seen coming out of stars, all are either $\pi$-mesons or protons. No $\mu$-mesons are seen, and no electrons.

Other observations reported by Prof. Powell were the following. (i) 500 examples of $\pi-\mu$ decay observed at the Jungfraujoch were analysed to see whether the $\mu$-tracks showed any directional asymmetry, such as Wentzel has suggested might occur if the $\pi$-meson has a spin, in view of the predominantly vertical orientation of the $\pi$-producing nucleon beam. No asymmetry was detectable, a result which, of course, does not exclude the possibility of a nonzero spin for the $\pi$-meson. (ii) Many tracks have been measured of electrons with energies greater than $100 \mathrm{MeV}$., and all have grain densities less than 1.1 times minimum; thus the theoretically predicted relativistic increase of ionization with energy does not appear to exist. Dr. J. G. Wilson suggested an explanation: the relativistic increase of ionization is supposed to be due to atoms at large distances from the moving particle, in a region where ions will be formed only sparsely and with small energies; thus if the photographic grains respond not to single ions but to groups of ions close together, the extra ionization produced by a highly relativistic particle will not produce a corresponding increase in grain density. (iii) Using an analysis of small-angle scattering, King at Bristol has measured the energies of electron-positron pairs which are generated in plates at high altitudes by photons of the soft component; in a large stack of plates, the frequency of such pairs increases with depth in the stack, so that a considerable proportion of the originating photons are locally generated, and it may be presumed that these photons arise in part from nuclear collisions in the upper layers of the stack. The observed energy distribution of the pairs has a maximum at about $100 \mathrm{MeV}$., falls off rather steeply below this maximum and more gradually above it. Such a distribution is pleasantly suggestive of a neutral meson of mass 300 , which is created in nuclear collisions and decays at once into two photons. However, these observations are still at a preliminary stage. (iv) Numerous examples have been found of the so-called 'giraffe' events (nomenclature due to Occhialini) in which one relativistic track suddenly splits into three relativistic tracks with very small angular divergence. From scattering measurements, it is found that there is a rough energy-balance between the one ingoing and the three outgoing tracks; also the rest-masses of all particles involved are of the order of the electron mass. The frequency of these events seems much too great for them to be explained as pair-creations due to an electron passing through the field of an atom. They remain a mystery, about which it is hoped more will be heard.

In the theoretical section of the programme there were three talks describing the work of the Edinburgh school, one by Prof. Max Born himself, one by Dr. L. M. Yang and one by Dr. Kai-Chia Cheng. These talks gave a general account of the reciprocity theory of elementary particles which Prof. Born has origin. ated. The theory aims at providing, at one blow, a consistent formalism free of divergences for the description of quantized fields, and an explanation of the various types of existing particles and their masses. Since the theory is yet by no mears in a complete form, the conference was not in a position to pass judgment upon it. Because of its technical mathematical character, no attempt will be made to summarize it here.

Prof. Niels Bohr gave a talk on the theory of elementary particles, in which he described the ideas and methods which were followed in the analysis of the concepts of quantum electrodynamics which he has recently completed with Prof. L. Rosenfeld. The result of this analysis has been to show that the concepts of quantum electrodynamics form a logically consistent and intelligible framework. This makes the future task of the theoretical physicist more difficult rather than easier, because it is now clear that 
the real problems concerning the nature and existence of elementary particles cannot be met and attacked within the domain of electrodynamics.

Other theoretical papers were read by Prof. F. Bopp of Munich, Prof. L. Jánossy of Dublin, Prof. M. Fierz of Basle, and by Sir Charles Darwin. Prof. Bopp presented a new method for formulating the dynamics of particles interacting with an electromagnetic field, when the Lagrangian of the system involves not only particle velocities but also accelerations; he is able to quantize this formalism, and to obtain from it various elegant consequences; however, the quantized theory is not yet completely worked out and adapted for practical use.

At the end of the theoretical programme, Sir Charles Darwin put forward some radical speculations concerning the origin of cosmic rays, which aroused widespread discussion. He first proposed for consideration the phenomenon of pitting of marine propeller-blades as a result of cavitation at the surface of the blades; in this phenomenon a small hemispherical eavity in the water is supposed to collapse in a symmetrical manner, and it follows from the laws of hydrodynamies that a very small mass of water in contact with the blade will arrive at the centre of the cavity at the instant of collapse with an immensely high velocity. He suggested that the origin of cosmic rays be sought, not in any thermodynamically stable process, but in some violently non-thermodynamical and co-operative phenomenon such as he had described. Pushing a little farther the analogy with cavitation, he proposed for investigation the possibility that cosmic rays could be generated in the collapse of large vacuous regions formed by the turbulent motions in stellar atmospheres [seo also Nature, December 31, p. 1112]. Sir George Thomson, however, pointed out that because of the well-known order of magnitude of the cosmic-ray flux, either the generation process in stars must be unreasonably efficient, or else the generation must be taking place in our own sun.

Present at the conference were about a hundred physicists, representatives of ten countries. Russian physicists, who also had been invited to attend, were absent. However, at one of the sessions Prof. Born read a telegram which he had received from Prof. Vavilov, president of the Academy of Sciences of the U.S.S.R., apologizing for not sending a delegate and expressing friendly interest in the proceedings of the conference.

\section{OBITUARIES}

\section{Prof. D. R. Hoagland}

THE death of Dennis Robert Hoagland, professor of plant nutrition in the University of California, Berkeley, has removed an illustrious plant physiologist and one of the world's leading authorities in the broad field of plant and soil interrelations. For more than thirty years he laboured with distinction and devotion toward the understanding of the extremely complex plant-soil-atmosphere system as it exists in Nature.

Hoagland was born in Golden, Colorado, and received his early education in Denver. In 1903 he went to study chemistry at Stanford University. Following a distinguished undergraduate career he first elected to go on with advanced work in chemistry; but in 1908 he accepted a position in the Laboratory of Animal Nutrition in the University of
California. The new position brought him for the first time in professional contact with biology and took him to Berkeley, where, except for a few short gaps, he was to spend the rest of his life.

Hoagland remained in the field of animal nutrition for five years, the last of which he spent in research under Prof. E. V. McCollum, of the University of Wisconsin. In later years, Hoagland looked back on his association with Prof. McCollum as his real inspiration for a life devoted to scientific research. There would normally have been every expectation that he would continue in this field, but circumstances intervened and the acceptance in 1913 of a position as assistant professor of agricultural chemistry brought him back to Berkeley and was the initiation of his work in plant nutrition.

One of his early research activities concerned itself with the effect of hydrogen-ion concentration on plant growth. It was with great surprise that he discovered that, contrary to the then accepted tenets of agricultural teaching, barley plants made excellent growth at $p H$ 5. It was apparent that the inherent complexity of soils rendered untrustworthy many sweeping generalizations based on local conditions. He became convinced that the complex problem of soil and plant interrelations could best be studied by techniques which permitted rigid experimental control and the isolation of individual variables. He afterwards developed to a very high degree the water-culture technique for growing plants which has since, for more than three decades, served as a powerful and discriminating tool for studying fundamental problems in plant physiology at Berkeley. In later years he was also instrumental in securing one of the first experimental chambers in the United States for the growing of plants under reproducible conditions of light, temperature and humidity.

His early and dominant research interest was in the process of absorption and accumulation of ions by plants. The quality of the immense contribution which he, with his collaborators and associates, made to this fundamental process of plant physiology can only be judged against a background of the ideas and concepts which prevailed when his work got under way. There was no clear view as to how inorganic elements are absorbed by plants. Discussions of this subject were usually invoking such concepts as osmosis, permeability, antagonism, none of which, as he later clearly demonstrated, could explain their absorption, accumulation and retention against a concentration gradient of anions and cations by plant cells.

Hoagland first became interested in the special nature of ion absorption by plants during the First World War, when during a search for domestic sources of potassium, he discovered the remarkable ability of the giant kelps of the Pacific Coast to accumulate potassium and iodine many times in excess of the concentrations found in the sea. He returned to this problem several years later, using first intact barley plants and, later, a unicellular freshwater alga, Nitella. This work clearly demonstrated that absorption of ions by plants is a metabolic process requiring energy and not a question of simple permeability. His work with Nitella allowed him to paint in bold strokes a theory which at once correlated a host of observations in the field and laboratory and made it possible to approach an infinitely complex subject with principles instead of mere empirical observations. The next phase of his researches on 